\title{
INHALTSÜB ERSICHT
}

Vorwort ........................... X XI

Abkürzungs- und Siglenverzeichnis . . . . . . . . . . . . . . . . . XV

Zur Benutzung . . . . . . . . . . . . . . . . . . . . XXIV

439. Jakob Philipp Hackert [Ankündigung und Auszug] . . . . . . . . . . . . . . . 1

440. Philipp Hackert . . . . . . . . . . . . . . . . . . . . . . . . . . . . . . 3

441. Hackert: Zwei Landschaften von Philipp Hackert . . . . . . . . . . . . . . . 57

442. Hagen: Olfried und Lisena, ein romantisches Gedicht in zehn Gesängen ...

[Ankündigung] . . . . . . . . . . . . . . . . . . . . . . . . . . . . . 63

443. Hagen: Olfried und Lisena. Ein romantisches Gedicht in zehn Gesängen ... . . . 63

444. Hagen: Olfried und Lisena noch einmal . . . . . . . . . . . . . . . . . . 71

Hagen, v. d.: Tausend und ein Tag. Morgenländische Erzählungen, nach von der Hagens Übersetzung ... siehe: Tausend und ein Tag.

Haller: Briefe über die wichtigsten Wahrheiten der Offenbarung siehe: Frankfurter gelehrte Anzeigen (EGW 6, 105).

Haller: Usong, eine Morgenländische Geschichte in vier Büchern siehe: Frankfurter gelehrte Anzeigen (EGW 6, 106).

445. Hamann . . . . . . . . . . . . . . . . . . . . . . . . . . . . . . . . . 80

Hamburger Vorspiel siehe: Schema zu einem Vorspiel bey Eröffnung des neuen Hamburger Theaters.

446. Hanau [Nachtrag] . . . . . . . . . . . . . . . . . . . . . . . . . . . . . . 87

447. Handschriften . . . . . . . . . . . . . . . . . . . . . . . . . . . . . . . 90

Handzeichnungen siehe: Über die Entstehung der zweiundzwanzig Blätter meiner Handzeichnungen (EGW 4, 2).

Handzeichnungen Goethes siehe: Schwerdtgeburth: Radierte Blätter ...

Hans der Schuflicker; Die Pilgrimme von Mecca; Röschen und Colas; der Zauberer ... siehe: Frankfurter gelehrte Anzeigen, Poinsinet (EGW 6, 118).

448. Hanswursts Hochzeit oder der Lauf der Welt . . . . . . . . . . . . . . . . . 97

449. Harmonie der Farben . . . . . . . . . . . . . . . . . . . . . . . . . . . 104

Hasche: Vermischtes Magazin eine Wochenschrift ... siehe: Frankfurter gelehrte Anzeigen (EGW 6, 106).

450. Der Hausball. Eine deutsche Nationalgeschichte . . . . . . . . . . . . . . . . 106

Hausen: Leben und Charakter Herrn Christian Adolph Klotzens ... siehe:

Frankfurter gelehrte Anzeigen (EGW 6, 106). 
451. Hausmanns Vorlesung . . . . . . . . . . . . . . . . . . . . . . . . . 108

452. Joseph Haydns Schöpfung. Aufgeführt an dessen Geburtstage dem 31. März 1826109

453. Hebel: Allemannische Gedichte. Für Freunde ländlicher Natur und Sitten . . . . 111

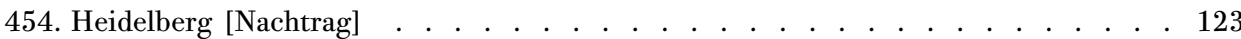

455. Die heiligen drei Könige I. Manuscript, lateinisch, aus dem funfzehnten Jahrhundert 126

456. Die heiligen drei Könige II. Auf Seite 156 [169] bezüglich . . . . . . . . . . . . 128

457. Die heiligen drei Könige III . . . . . . . . . . . . . . . . . . . . . . 136

458. Die heiligen drei Könige noch einmal . . . . . . . . . . . . . . . . . . . . 140

Über Heinrich Füeßli's Arbeiten siehe: Füeßli (EGW 6, 167).

Heinrich Meyer's Tabelle zu dessen Kunstgeschichte siehe: Meyer.

459. Heinroths Anthropologie . . . . . . . . . . . . . . . . . . . . . . . . . . 146

Helena classisch-romantische Phantasmagorie siehe: Faust (EGW 5).

460. Helena in Edinburgh, Paris und Moskau . . . . . . . . . . . . . . . 148

Helvig: Frithiof siehe: Vorläufige Anzeige

461. Hemsterhuis-Galizinische Gemmen-Sammlung . . . . . . . . . . . . . . . . . 157

462. Henschel: Physiognomische Skizzen der Gebrüder Henschel . . . . . . . . . . 177

463. Herder . . . . . . . . . . . . . . . . . . . . . . . . . . . . . 179

464. Herder: Idées sur la philosophie de l'histoire de l'humanité par Herder, traduites par Quinet. Paris 1828 . . . . . . . . . . . . . . . . . . . . 190

465. Hermann: Die tragischen Tetralogien der Griechen, Programm von Ritter Hermann. 1819 . . . . . . . . . . . . . . . . . . . 198

466. Hermann und Dorothea . . . . . . . . . . . . . . . . . . . . . . . . . 202

Heroische Statuen von Tieck siehe: Tieck, Fr.

467. Herr Mawe. Nachricht von seinen letzten Expeditionen im Oktober 1817 . . . . 319

Des Herrn G. R. von Göthe sämmtliche Werke in acht Bänden siehe: Werke, Ankündigung der Ausgabe S.

Herrn Hollands philosophische Anmerkungen über das System der Natur ... siehe: Frankfurter gelehrte Anzeigen (EGW 6, 106).

468. Herrn von Hoffs geologisches Werk . . . . . . . . . . . . . . . . . . . 328

469. Herschels Beobachtungen ... Wirkung farbiger Beleuchtung auf Pflanzen . . . . 331

Herstellung des Straßburger Münster siehe: Alt-Deutsche Baukunst (EGW 1, 25).

470. Hervortreten des Unterschiednen . . . . . . . . . . . . . . . . . . . . . . 338

Herwich: Franken zur griechischen Litteratur ... siehe: Frankfurter gelehrte Anzeigen (EGW 6, 107).

Herwich: Nachricht [über J. J. Herwichs Vorlesungen über die elegante Literatur; Voranzeige] siehe: Frankfurter gelehrte Anzeigen, Nachricht (EGW 6, 117).

Herwig: Wahre Beschreibung zweyer aneinander gewachsener Kinder siehe: Frankfurter gelehrte Anzeigen (EGW 6, 107).

471. Herzogliches Hoftheater zu Weimar 1792 . . . . . . . . . . . . . . . . . . . 339

472. Herzogliches Hoftheater zu Weimar 1795 . . . . . . . . . . . . . . . . . . . 342

v. Heufeld: Theateralmanach für das Jahr 1773, verfasset von einigen Liebhabern der deutschen Schaubühne ... siehe: Frankfurter gelehrte Anzeigen (EGW 6, 107).

Heyne: Einleitung in das Studium der Antike, oder Grundriß einer Anführung zur Kenntniß der alten Kunstwerke ... siehe: Frankfurter gelehrte Anzeigen (EGW $6,107)$. 
473. Gottlieb Hillers Gedichte und Selbstbiographie. Erster Theil. 1805 . . . . . . . 344

474. Über die Hindernisse, die dem modernen Künstler im Wege stehen, vom gestaltlosen zur Gestalt zu gelangen . . . . . . . . . . . . . . . . . 347

475. Hinrichs: Das Wesen der antiken Tragödie, in ästhetischen Vorlesungen durchgeführt von Hinrichs . . . . . . . . . . . . . . . . . . . . . . . . . 352

476. Über Aloys Hirt . . . . . . . . . . . . . . . . . . . . . . . . . . . . . 357

Histoire de la vie et des ouvrages de Molière siehe: Taschereau.

477. Hittdorf et Zanth: Architecture antique de la Sicile . . . . . . . . . . . . . . 360

478. Hittdorf et Zahnt: Architecture moderne de la Sicile . . . . . . . . . . . . . . 362 Hochländisch siehe: Übersetzungen.

479. Über den Hochschnitt . . . . . . . . . . . . . . . . . . . . . . . . . . . 363

Hoff siehe: Herrn von Hoffs geologisches Werk (S. 328).

Holland siehe: Herrn Hollands philosophische Anmerkungen über das System der Natur ... siehe: Frankfurter gelehrte Anzeigen (EGW 6, 106).

480. Höhen der alten und neuen Welt bildlich verglichen . . . . . . . . . . . . . 377

481. Höherer Chemismus des Elementaren . . . . . . . . . . . . . . . . . . . . 385

482. Hör-, Schreib- und Druckfehler . . . . . . . . . . . . . . . . . . . . . . 387

483. Über das Hofleben . . . . . . . . . . . . . . . . . . . . . . . . . . . . . 389

484. Hoftheater zu Weimar . . . . . . . . . . . . . . . . . . . . . . 391

Das Hohelied Salomons siehe: Übersetzungen.

v. Holbach: Examen de l'Essay sur les préjugés. Par le Philosophe de Sans-Souci.

London 1772 siehe: Frankfurter gelehrte Anzeigen (EGW 6, 108).

485. Holtei: Gedichte in schlesischer Mundart . . . . . . . . . . . . . . . . . . . 404

Homer siehe: Benjamin Constant über Ilias und Odyssee (EGW 2, 232).

siehe: Einleitung zu Ilias im Auszug (EGW 3, 273f.).

siehe: Aus Homer.

siehe: Homer nach Antiken gezeichnet, achtes Heft (S. 408).

siehe: Homer nach Antiken, neuntes Heft (S. 410).

siehe: Homer noch einmal (S. 412).

siehe: Homers Apotheose (S. 424).

siehe: Aus Homers Odyssee. Siebenter Gesang, Vers 78 bis 131.

siehe: Iliadis Fragmenta (S. 491).

siehe: Ilias im Auszug (S. 493).

siehe: Ilias, in Prosa übersetzt von Zauper, Odyssee, freie Nachbildung in zehnzeiligen Reimstrophen von Hedwig Hülle (S. 517).

siehe: Versuch, eine Homerische dunkle Stelle zu erklären.

Aus Homer siehe: Übersetzungen.

486. Homer nach Antiken gezeichnet, achtes Heft . . . . . . . . . . . . . . . . . 408

487. Homer nach Antiken. Neuntes Heft . . . . . . . . . . . . . . . . . . . . 410

488. Homer noch einmal . . . . . . . . . . . . . . . . . . . . . . . . . . . . 412

489. Homers Apotheose . . . . . . . . . . . . . . . . . . . . . . . . . . . . . 424

Aus Homers Odyssee. Siebenter Gesang, Vers 78 bis 131 siehe: Übersetzungen.

Des Hommes Célèbres de France au dixhuitième siècle ... siehe: [Diderot III] (EGW $3,28)$.

490. Hood: Whims and Oddities . . . . . . . . . . . . . . . . . . 430 
491. Von dem Hopfen und dessen Krankheit, Ruß genannt 430

Hoppe: Joachimi Hoppii Commentatio succincta ad Institutiones justinianeas ... siehe: Frankfurter gelehrte Anzeigen (EGW 6, 108).

Horazens Oden von [K. A.] Kütner siehe: Frankfurter gelehrte Anzeigen, Kütner (EGW 6, 110).

492. Der Horn

493. Hornblendekugel bei Weimar . . . . . . . . . . . . . . . . . . . . 440

Howard siehe: Goethe zu Howard's Ehren (EGW 6, 613).

siehe: Luke Howard an Goethe.

siehe: Luke Howard to Goethe. A biographical Scetch (S. 441).

siehe: Wolkengestalt nach Howard.

Luke Howard an Goethe siehe: Übersetzungen.

494. Luke Howard to Goethe. A biographical Scetch

Hülle: siehe: Ilias, in Prosa übersetzt von Zauper, Odyssee, freie Nachbildung in zehnzeiligen Reimstrophen von Hedwig Hülle (S. 517).

495. Humboldt, A. v.: Über den Bau und die Wirkungsart der Vulkane [I] . . . . . . 442

496. Humboldt, A. v.: Über den Bau und die Wirkungsart der Vulkane [II] . . . . . . 445

497. Humboldt, A. v.: Berlin: Ideen zu einer Physiognomik der Gewächse . . . . . . 448

498. Hundeshagen: Kaiser Friedrich I. Barbarossa Palast in der Burg Gelnhausen. Von

Bernhard Hundeshagen. Mit 13 Kupferabdrücken ... . . . . . . . . . . . . . 452

Hunger: Thrasybulus. Oder von der Liebe zum Vaterlande siehe: Frankfurter

gelehrte Anzeigen (EGW 6, 108).

Ich auch ... siehe: Übersetzungen.

Ich glaubte nicht ... siehe: Übersetzungen.

499. Ideen über organische Bildung [Plan]

Ideen zu einer Physiognomik der Gewächse siehe: Humboldt, A. v. (S. 448).

Idées sur la philosophie de l'histoire de l'humanité par Herder, traduites par

Quinet. Paris 1828 siehe: Herder, Idées (S. 190).

Idylle siehe: Maskenzüge. Idyllische Kantate zum 30. Januar 1813.

Iffland siehe auch: Über die Entstehung des Festspiels zu Ifflands Andenken (EGW $4,3)$.

siehe auch: Nachspiel zu Ifflands Hagestolzen.

siehe auch: Zu Schillers und Ifflands Andenken.

500. Iffland: Almanach für Theater und Theaterfreunde, auf das Jahr 1807. Von August Wilhelm Iffland . . . . . . . . . . . . . . . . . . . . . . . . . . . . 474

501. Besuch von Iffland, auf meiner Reise über Mannheim nach der Schweiz im Jahre 1779 . . . . . . . . . . . . . . . . . . . 475

502. Das Igeler Monument . . . . . . . . . . . . . . . . . . . 477

503. Iken: Eunomia von Dr. Karl Iken. Drei Bände. Grimma 1827 . . . . . . . . . 483

504. Iken: Leukothea von Dr. Karl Iken. Leipzig 1827. 2 Bände . . . . . . . . . . 490

Iken: Touti Nameh siehe: Touti Nameh.

Il conte di Carmagnola siehe: Manzoni.

505. Iliadis Fragmenta . . . . . . . . . . . . . . . . . . . . . 491

506. Ilias im Auszug . . . . . . . . . . . . . . . . . . . . . . . . . . 493

507. Ilias, in Prosa übersetzt von Zauper, Odyssee, freie Nachbildung in zehnzeiligen Reimstrophen von Hedwig Hülle . . . . . . . . . . . . . . . . . . . . 517 
508. In Sachen der Physik contra Physik . . . . . . . . . . . . . . . . 526

509. In wiefern die Idee: Schönheit sei Vollkommenheit mit Freiheit, auf organische Naturen angewendet werden könne . . . . . . . . . . . . . . . . . 530

510. Über die Incommunicabilien unter den Paralipomenen . . . . . . . . . . . . . 533 Indicazione di ciò che nel 1819 si è fatto in Italia intorno alle lettere, alle scienze ed alle arti siehe: Übersetzungen

511. Indische Dichtungen . . . . . . . . . . . . . . . . . . . . . . . . . . . . 537

Anhang zum Artikel Nr. 484 Hoftheater zu Weimar . . . . . . . . . . . . 545

Abbildungen . . . . . . . . . . . . . . . . . . . . . . . . . . . 547 
